LA-UR-01-3828

Approved for public release; distribution is unlimited.

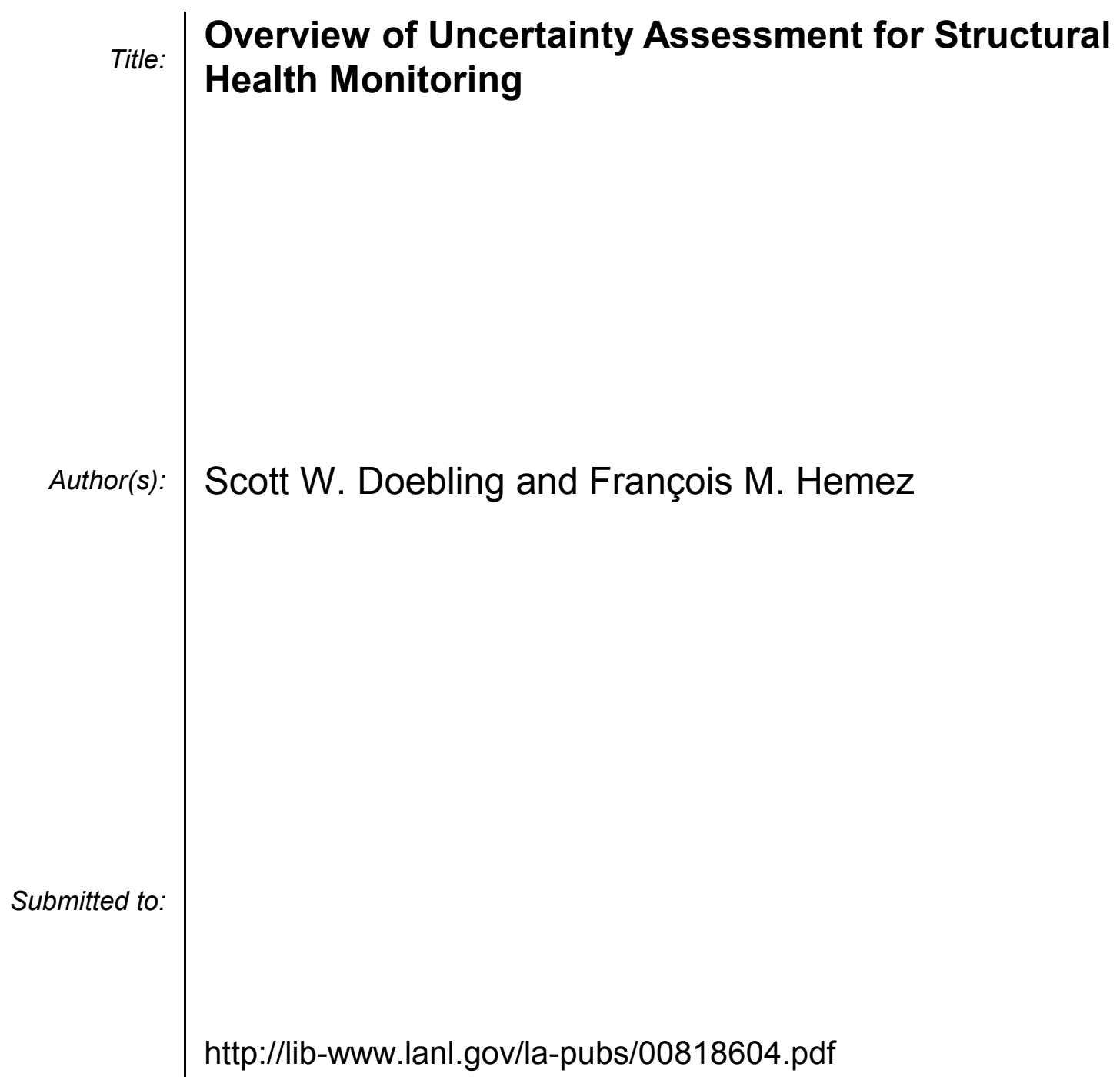

Los Alamos National Laboratory, an affirmative action/equal opportunity employer, is operated by the University of California for the U.S. Department of Energy under contract W-7405-ENG-36. By acceptance of this article, the publisher recognizes that the U.S. Government retains a nonexclusive, royaltyfree license to publish or reproduce the published form of this contribution, or to allow others to do so, for U.S. Government purposes. Los Alamos National Laboratory requests that the publisher identify this article as work performed under the auspices of the U.S. Department of Energy. Los Alamos National Laboratory strongly supports academic freedom and a researcher's right to publish; as an institution, however, the Laboratory does not endorse the viewpoint of a publication or guarantee its technical correctness. 


\title{
Overview of Uncertainty Assessment for Structural Health Monitoring
}

\author{
Scott W. Doebling and François M. Hemez \\ Engineering Analysis Group (ESA-EA) \\ Los Alamos National Laboratory \\ P.O. Box 1663, M/S P-946, Los Alamos, New Mexico 87545
}

\begin{abstract}
Uncertainty quantification is an emergent field in engineering mechanics that makes use of statistical sampling, hypothesis testing and input-output effect analysis to characterize the effect that parametric and non-parametric uncertainty has on physical experiment or numerical simulation output. This publication overviews a project at Los Alamos National Laboratory that aims at developing a methodology for quantifying uncertainty and assessing the total predictability of structural dynamics simulations. The propagation of parametric variability through numerical simulations is discussed. Uncertainty assessment is also a critical component of model validation, where the total error between physical observation and model prediction must be characterized. The purpose of model validation is to assess the extent to which a model is an appropriate representation of reality, given the purpose intended for the numerical simulation and its domain of applicability. The discussion is illustrated with component-level and system-level validation experiments that feature the response of nonlinear models to impulse excitation sources. This publication is unclassified; it is approved for unlimited, public release (number LA-UR-01-3828).
\end{abstract}

\section{INTRODUCTION}

The availability of Tera-Ops computing where speeds of $10^{+12}$ multiplications per second are exceeded; general purpose finite element modeling and analysis packages; and advances in computer graphics promote the development of ever-increasingly complex computer simulations [1]. In the field of structural dynamics, applications such as structural health monitoring and damage assessment are evolving into system-level analysis techniques (as opposed to component-level). For example, the vibration response of an entire bridge is simulated without relying on model condensation techniques that approximate the geometry or reduce the number of degrees of freedom [2]. Similarly,

Scott W. Doebling, Los Alamos National Laboratory, Engineering Analysis Group (ESA-EA), M/S P946, Los Alamos, New Mexico 87545, doebling@lanl.gov (E-mail), 505-667-6950 (Voice).

François M. Hemez, Los Alamos National Laboratory, Engineering Analysis Group (ESA-EA), M/S P946, Los Alamos, New Mexico 87545, hemez@lanl.gov (E-mail), 505-665-7955 (Voice). 
numerical simulations for health monitoring are increasingly modeling the sources of nonlinearity that may contribute to the system's response. An example is the transient response of suspension and cable-stayed bridges that induces complex energy dissipation phenomena [3].

This evolution, however, leads to new problems. First, conventional features such as resonant frequencies and mode shapes become obsolete when the response of the system exhibits nonlinear and non-stationary components. New strategies must be devised for test-analysis correlation, finite element model updating and parameter calibration. Uncertainty quantification and propagation of variability are additional difficulties that the conventional forward and inverse problem solving technologies do generally not address. Consequently, most approaches available for structural health monitoring are restricted to systems where non-linearity and time dependency are "small and regular" enough that first-order, linear approximations remain acceptable [4]. The frequency-domain formulation and modal transform of the equations of motion are typical examples of firstorder approximation.

\subsection{Structural Health Monitoring Research at Los Alamos}

To address these difficulties, several research and development efforts have been initiated at Los Alamos National Laboratory (LANL). The four aspects that contribute to the technology being developed can be categorized as:

\section{(A) Sensing Capabilities;}

(C) Uncertainty Quantification;

\section{(B) Data Interrogation;}

(D) Structural Prognosis.

The first aspect (instrumentation) focuses on the development of wireless, decentralized sensors that also feature localized computing capabilities. This goal is achievable given the recent advances in the semi-conductor and MEMS technology. By deploying an array of small, non-intrusive and inexpensive sensors and by letting them process the data locally, the amount of communication required between the various sensing stations is reduced and decision making is greatly facilitated.

The second aspect investigated (data interrogation) involves the reduction of the vast amounts of data collected and generated by continuous monitoring and numerical simulation. Data condensation and feature extraction tools are developed in the general context of statistical pattern recognition. A particular emphasis is placed on developing features that enable the analysis of nonlinear and non-stationary responses. Another critical issue is data normalization, which consists in ensuring that vibration changes observed from one data set to another result from changes in the structural condition as opposed to changes in loading, response levels and environmental condition [5]. 
The third aspect is the quantification of uncertainty and the validation of numerical models for complex engineering simulations. Statistical methods for the design of physical or computer experiments are investigated to identify which input parameters are responsible for the response's variability and to study the interaction between inputs and outputs. Once this is understood, surrogate models can be developed for efficient numerical optimization, damage detection and prognosis. The tools developed for uncertainty assessment can be applied to test data sets and numerical models that feature arbitrary material non-linearity, contact mechanics and transient excitation sources [6].

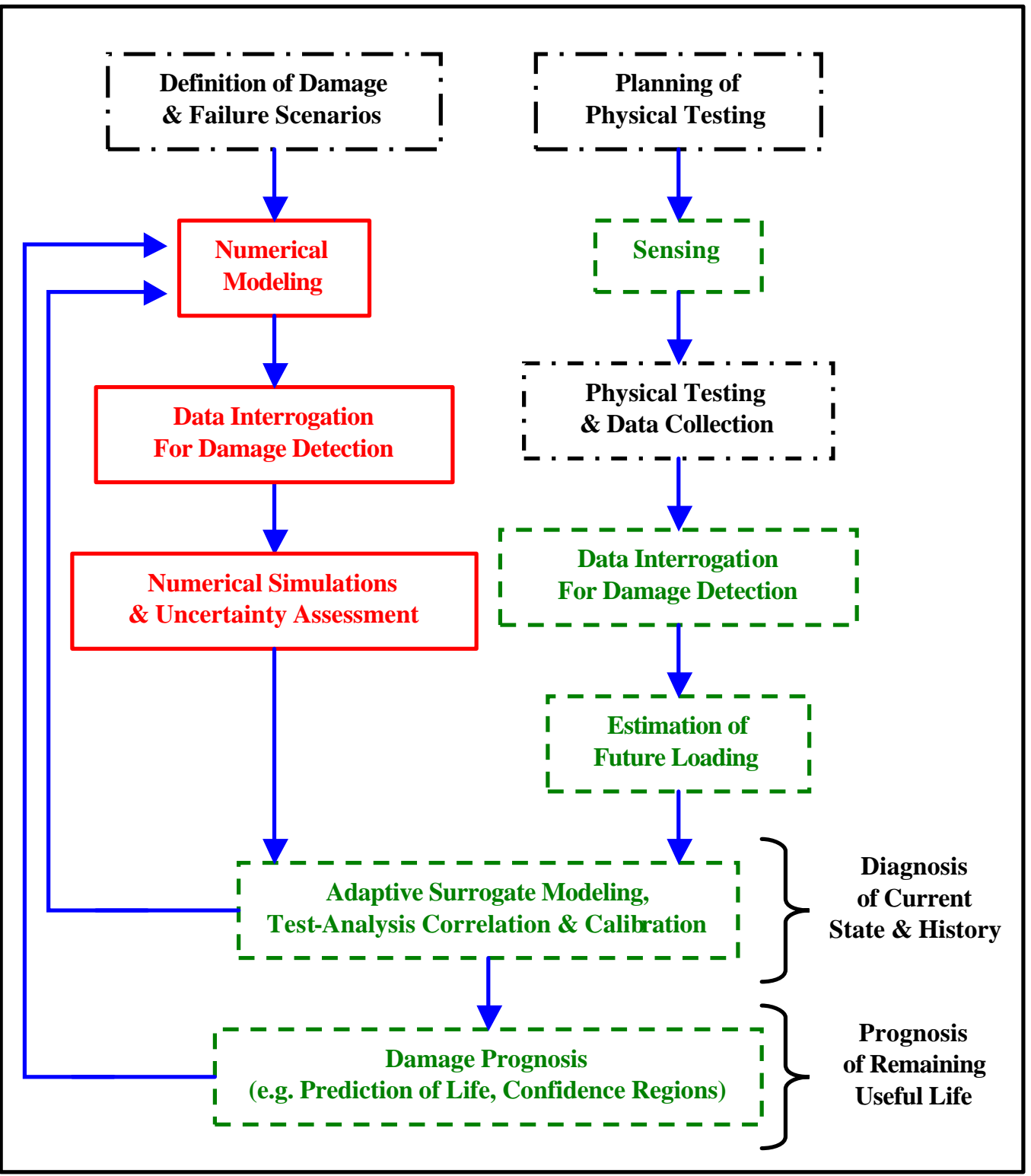

FIGURE 1. Flow-chart of Integrated Structural Health Monitoring. (Black, dashed-dotted: Planning and data acquisition. Red, solid: Large-scale modeling and computing. Green, dashed: On-board integration.) 
The last step (structural prognosis) refers to the assessment of system performance given its current structural condition and past history. A critical issue in structural prognosis is the identification of future operating conditions and loading. Uncertainty quantification and plays, again, a central role because uncertain future loads must be estimated and propagated through the surrogate models within a given degree of confidence. Structural prognosis also includes decision-making to help the analysts and users balance performance and risk assessment.

Figure 1 illustrates the methodology for structural health monitoring developed at Los Alamos. It can be observed that the four aspects (A-D) contribute to many of the steps outlined in Figure 1. Although it is our opinion that the successful deployment of damage detection technology at the system level will require all four aspects to be addressed, this publication focuses on the uncertainty assessment (C) only. In the remainder, the forward propagation of uncertainty is dscussed and illustrated with the numerical simulation of Taylor material testing. Then, an example of system-level uncertainty assessment is provided with the numerical simulation of an impulse through a complicated threaded assembly. This example illustrates the analysis of input-output relationship in the case of a large-scale finite element simulation. Finally, the publication is concluded with a discussion of total uncertainty assessment.

\section{FORWARD PROPAGATION OF UNCERTAINTY}

The goal of uncertainty quantification is to characterize the effect that parametric variability and non-parametric uncertainty have on physical experiment or numerical simulation output. It is emphasized that "uncertainty" is not necessarily restricted to parametric uncertainty, that is, the imperfect knowledge of the control parameters of a physical experiment or input parameters of a numerical model. Uncertainty may also take the form of stochastic equations of motion, environmental variability, measurement errors, discretization and numerical errors, to name only a few.

Statistical sciences provide many tools for the efficient sampling of probability information, hypothesis testing and investigation of the interaction between inputs and outputs. An example of international forum where the latest advances in these techniques are discussed is the SAMO (Sensitivity Analysis of Model Output) conference [7]. Unfortunately, application to engineering mechanics problems remains, to this date, somewhat limited.

In the following, uncertainty in a quantity is described in terms of a Probability Density Function (PDF) that specifies the probability of all possible values of that quantify. In this context, probability is used as the quantitative measure of the degree of belief, which summarizes the knowledge about a particular situation. Other mathematical frameworks are available for quantifying and propagating uncertainty that may offer attractive 
alternatives to the theory of probability, especially in the event of extreme uncertainty. Among them, we cite the Dempster-Shafer theory of possibility and belief [8], the theory of fuzzy sets [9], information gap theory and convex models of uncertainty [10]. For engineering applications, representing the uncertainty as probability information is reasonable because large amounts of test data and well-established formal theories are available.

The oldest and simplest method of uncertainty propagation is the Monte Carlo technique. The underlying idea is to randomly pick values of a parameter such that the histogram of chosen values approximates the PDF. Then, the computational model is analyzed at each point sampled in the input parameter space. Similarly, in the case of testing, a physical experiment is performed for each combination of sampled input parameters. For each analysis or test performed, the output feature is recorded. Finally, the distribution of output values is characterized with its histogram, that is, the frequency of occurrence of each value. If a large number of analyses are performed, the Central Limit Theorem establishes that the histogram of output values converges to the unknown but "true" distribution, hence, offering a complete characterization of the output variability. The main drawback of Monte Carlo sampling is that a large number of analyses is required, generally several thousand. It is therefore not practical if the analysis performed (experiment or simulation) is expensive or the input parameter space to explore is large. Other strategies are available that may achieve the same result with a smaller number of samples. Among them, we cite the Latin Hypercube sampling [11]; Taguchi sampling and other orthogonal array techniques [12]; and the Fourier Amplitude Sensitivity Test (FAST), based on a cyclic sampling strategy [13].

\subsection{Example: The Taylor Impact Test}

The Taylor impact test consists of impacting a cylindrical sample of material against a fixed, rigid surface. Taylor tests are often performed to investigate material behavior at high stress and high strain rates. Extremely high plastic strains develop at the crushed end of the rod, resulting in severe local deformation. The type of experimental measurements typically performed range from simply measuring the initial and final radii or lengths of the deformed cylinder, to a full specification of its profile. To illustrate the propagation of uncertainty using Monte Carlo sampling, a numerical simulation of the Taylor test is performed. The cylinder is made of a high-strength steel, $15 \mathrm{~mm}$ in diameter and $38-\mathrm{mm}$ long. The impact velocity is 350 meters-per-second. Because large plastic deformations and strain rates in excess of $10^{+5}$ second $^{-1}$ are expected, strain-rate dependent plasticity must be included in the model. The Johnson-Cook model for rate-dependent plasticity is adopted:

$$
\mathbf{s}=\left(\mathbf{a}_{1}+\mathbf{a}_{2} \mathbf{e}_{\mathbf{p}}^{\mathrm{N}}\right)\left(1+\mathbf{a}_{3} \log \left(\dot{\mathbf{e}}_{\mathbf{p}}\right)\right)
$$


where $\varepsilon_{\mathbf{p}}$ and $\sigma$ denote the equivalent plastic strain and resulting stress, respectively. The parameters $\alpha_{1}, \alpha_{2}, \alpha_{3}$ and $\mathbf{N}$ are material specific. The analysis of the cylinder's impact is performed with HKS/Abaqus, a general-purpose finite-element modeling package which employs explicit time integration [14]. Figure 2 illustrates the response obtained when the four material parameters $\alpha_{1}, \alpha_{2}, \alpha_{3}$ and $\mathbf{N}$ are set to their nominal values.

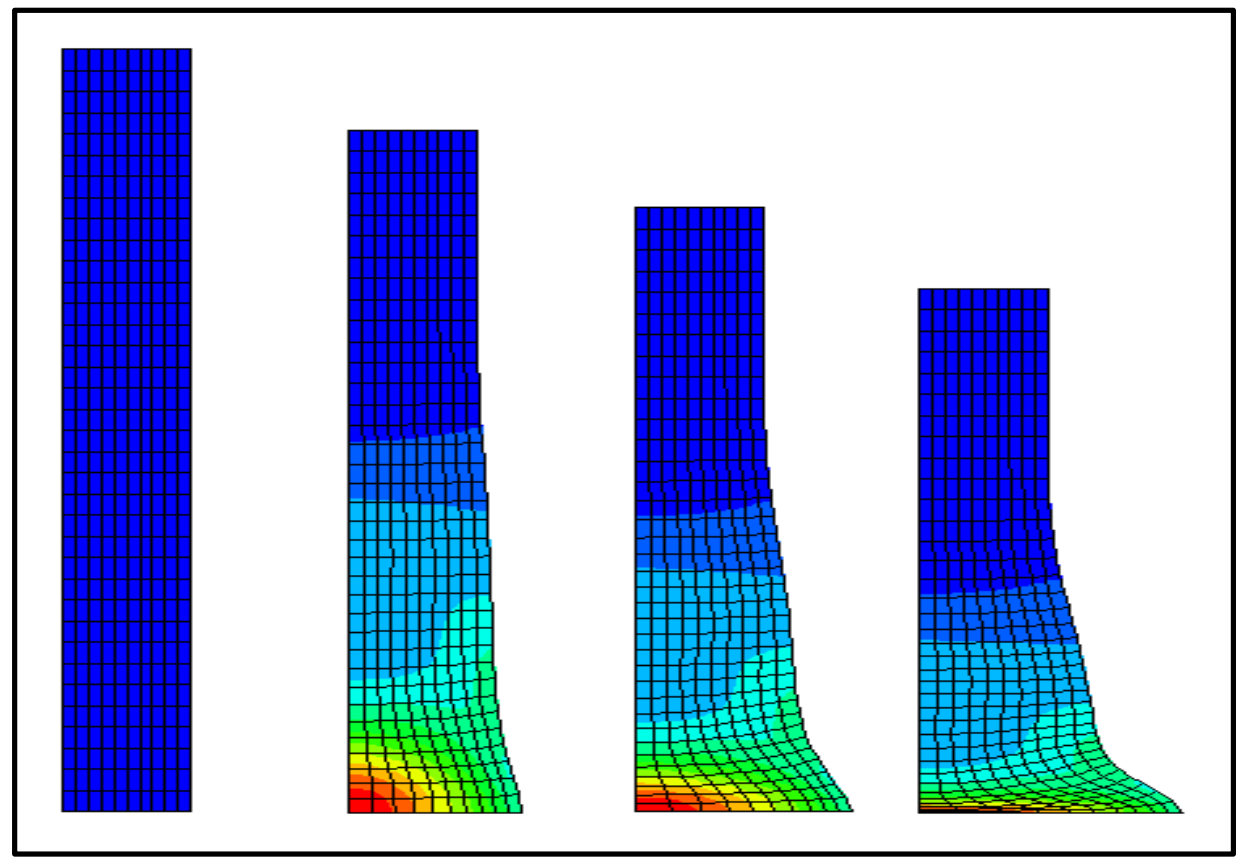

FIGURE 2. Simulation of the Taylor Test of a High-strength Steel.

(The response of an axisymmetric model is shown at times of $0 \mu \mathrm{s}, 17 \mu \mathrm{s}, 33 \mu \mathrm{s}$ and $50 \mu \mathrm{s}$ after impact, from left to right. The peak plastic strains for the three deformed shapes are equal to $83 \%, 165 \%$ and $248 \%$, respectively.)

The Monte Carlo technique is used to illustrate the forward propagation of uncertainty through the simulation code. We consider a hypothetical situation in which the only parameters that are uncertain are those in the above stress-strain model (1). It is further assumed that the uncertainty distribution in each parameter $\mathbf{p}_{\mathbf{j}}$ is independent and given by a normal distribution, $\mathbf{p}_{\mathbf{j}} \sim \mathbf{N}\left(\boldsymbol{\mu}_{\mathbf{j}} ; \mathbf{s}_{\mathbf{j}}\right)$. As indicated above, the Monte Carlo process consists of sampling parameter values from the assumed uncertainty distributions and running the simulation code for each set of values. This procedure for uncertainty propagation can therefore be viewed as nothing more than multiple runs of a deterministic computer program.

In this illustration, a total of 1,000 simulation runs are performed. Figure 3 shows the histograms of input parameters, that is, the frequency with which each value is sampled. It therefore represents the total variability inputted to the system. The two output features of interest are the ratios of final-to-initial cylinder lengths and radii, denoted by $\left(\mathbf{L} / \mathbf{L}_{\mathbf{0}}\right)$ and $\left(\mathbf{R} / \mathbf{R}_{\mathbf{o}}\right)$, respectively. Figure 4 illustrates the joint distribution of output features that results from input uncertainty. One noticeable observation is that the response parameters $\left(\mathbf{L} / \mathbf{L}_{\mathbf{0}}\right)$ 
and $\left(\mathbf{R} / \mathbf{R}_{\mathbf{o}}\right)$ are strongly correlated, as indicated by the non-circular distribution of points in the output feature space.
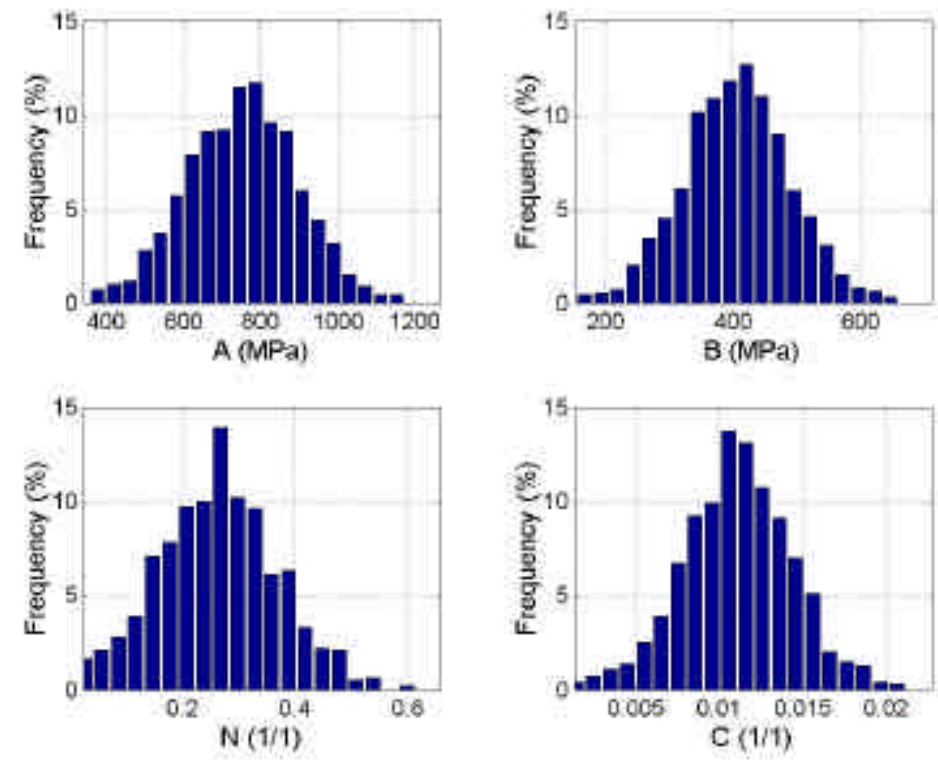

FIGURE 3. Histograms of Sampled Input Parameter Values.

(Upper left: Parameter $\alpha_{1}$. Upper right: Parameter $\alpha_{2}$. Lower left: Parameter $\boldsymbol{N}$. Lower right: Parameter $\alpha_{3}$. Symbols $\alpha_{1}, \alpha_{2}, N$ and $\alpha_{3}$ refer to equation (1).)

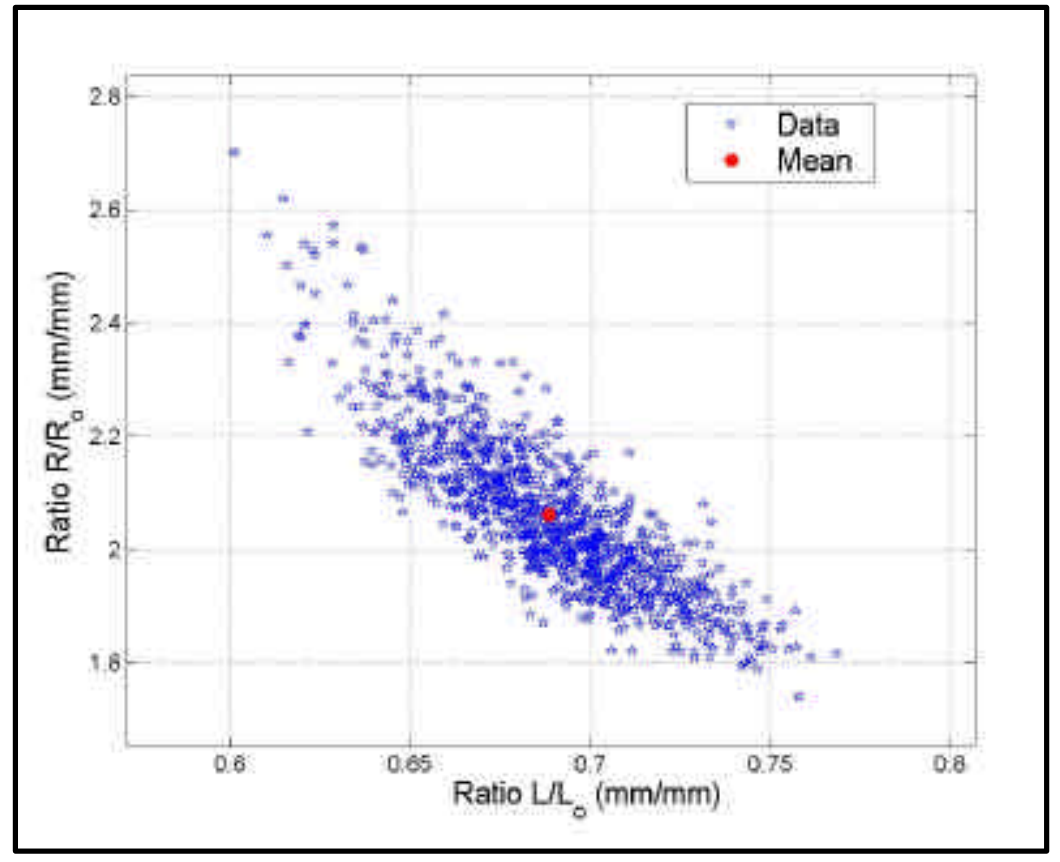

FIGURE 4. Distribution of Output Features $\left(L / L_{\mathbf{0}}\right)$ and $\left(R / R_{\mathbf{0}}\right)$.

(Horizontal axis: Ratios of final-to-initial lengths, $\left(\boldsymbol{L} \boldsymbol{L}_{o}\right)$. Vertical axis: Ratios of final-to-initial radii at the crushed end of the cylinder, $\left(\boldsymbol{R} / \boldsymbol{R}_{\boldsymbol{o}}\right)$.) 
Figure 5 shows the histograms of the ratios $\left(\mathbf{L}_{\mathbf{L}} \mathbf{L}_{\mathbf{0}}\right)$ and $\left(\mathbf{R} / \mathbf{R}_{\mathbf{0}}\right)$ obtained from the above joint distribution. In the case of the output feature $\left(\mathbf{R} / \mathbf{R}_{\mathbf{o}}\right)$, for example, the mean value of this distribution is equal to 2.06. Because the distribution represents the output uncertainty in $\left(\mathbf{R} / \mathbf{R}_{\mathbf{o}}\right)$, the RMS deviation of the distribution, $\mathbf{s}=0.14$, represents the estimate of the standard deviation in the uncertainty of our prediction for $\left(\mathbf{R} / \mathbf{R}_{\mathbf{0}}\right)$. It can further be observed that the two histograms shown in Figure 5 do not appear to be normally distributed, which should come as no surprise because the numerical simulation of this phenomenon is highly nonlinear.

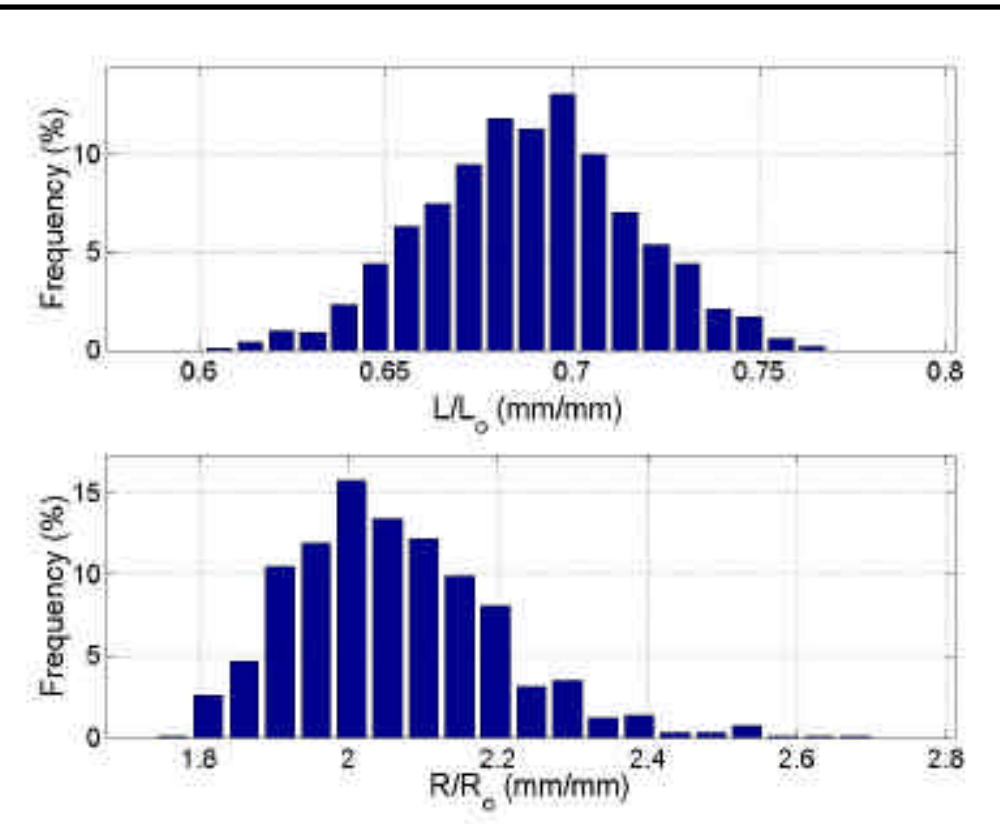

FIGURE 5. Histograms of Output Features $\left(L / L_{0}\right)$ and $\left(R / R_{0}\right)$.

(Top: Distribution of ratios of final-to-initial lengths, $\left(\boldsymbol{L}_{\mathbf{L}} \mathbf{L}_{\boldsymbol{o}}\right)$. Bottom: Distribution of ratios of final-to-initial radii at the crushed end of the cylinder, $\left(\boldsymbol{R} / \boldsymbol{R}_{\boldsymbol{o}}\right)$.)

For this example, the Monte Carlo calculation is handled using a scripting language that allows one to run a simulation code (such as HKS/Abaqus) with controlled input parameters and to summarize the output. The scripting language used here is Python [15]. Another possibility would be to use a commercial code such as NESSUS, which has been developed for reliability analysis of structural mechanics applications [16]. Other generalpurpose applications are being developed for the propagation of uncertainty, characterization of output probability information and inference concerning the parameter values. One example is the DAKOTA toolkit under development at Sandia National Laboratories [17]. 


\section{INPUT-OUTPUT EFFECT ANALYSIS}

One important application of uncertainty propagation is the analysis of input-output effects. Learning which combinations of input parameters are responsible for explaining the variability of the output is critical to understand complex physical experiments or numerical simulations. Effect analysis goes beyond conventional sensitivity analysis (based on differential sensitivity or approximation methods such as finite differences) because it assesses the sensitivity of the output over the entire range of applicability of the model. In contrast, local approaches only provide information in the neighborhood of a particular design point and a particular direction. Mathematically, effect analysis addresses the difficult problem of differentiating a function with respect to a random variable.

To illustrate the concept of effect analysis, consider that the phenomenon investigated (experiment or simulation) is replaced with a surrogate model:

$$
\mathbf{y}=\mathbf{a}_{\mathbf{o}}+\sum_{\mathrm{i}=1 \cdots \mathrm{N}} \mathbf{a}_{\mathrm{i}} \mathbf{p}_{\mathrm{i}}+\sum_{\mathrm{i}=1 \cdots \mathrm{N}} \sum_{\mathrm{j}=1 \cdots \mathrm{N}, \mathrm{j} \geq \mathrm{i}} \boldsymbol{\beta}_{\mathrm{ij}} \mathbf{p}_{\mathbf{i}} \mathbf{p}_{\mathbf{j}}
$$

Clearly, if all input parameters $\mathbf{p}_{\mathbf{j}}$ are normalized in $[-1 ;+1]$, then the relative magnitude between coefficients $\mathbf{a}_{\mathbf{j}}$ or $\mathbf{B}_{\mathbf{i j}}$ is indicative of the importance of the corresponding effect, whether it is a linear effect $\left(\mathbf{p}_{\mathbf{j}}\right)$, a linear interaction $\left(\mathbf{p}_{\mathbf{i}} \mathbf{p}_{\mathbf{j}}\right)$ or a quadratic effect $\left(\mathbf{p}_{\mathbf{j}}{ }^{2}\right)$. Statistical tests can be implemented to assess the global contribution of each effect to the total variability. Assume that the output variability can be explained by a particular input parameter or interaction effect denoted by $\mathbf{p}_{\mathbf{M}}$. The expected value of the output given $\mathbf{p}_{\mathbf{M}}$ is denoted by $\mathbf{y}=\mathbf{E}\left[\mathbf{y} \mid \mathbf{p}_{\mathrm{M}}\right]$. It can be shown that the total variance observed from the data is equal to the variance of $\mathbf{y}$ and the expected value of the total variance given $\mathbf{p}_{\mathbf{M}}$ :

$$
s^{2}(y)=s^{2}(y)+E\left[s^{2}\left(y \mid p_{M}\right)\right]
$$

Obviously, the last term in the right-hand side of equation (3) represents the influence of parameter effect $\mathbf{p}_{\mathbf{M}}$ on the total variability of the output $\mathbf{y}$. If this effect has little influence, then, the contribution $\mathbf{E}\left[\mathbf{s}^{2}\left(\mathbf{y} \mid \mathbf{p}_{\mathrm{M}}\right)\right]$ remains small. A popular statistic that approximates $\mathbf{E}\left[\mathbf{s}^{2}\left(\mathbf{y} \mid \mathbf{p}_{\mathrm{M}}\right)\right]$ is the R-square statistic $\left(\mathrm{R}^{2}\right)$. It is defined as the ratio of variance that can be attributed to a given effect to total variance:

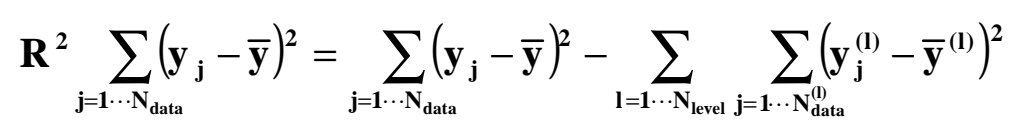

The procedure for input-output effect analysis consists of, first, designing a matrix of physical or computer experiments. The matrix provides the combination of input parameters $\mathbf{p}_{\mathbf{j}}$ at which the physical experiments or computer simulations are performed. The previous section 2 provides an example of uncertainty propagation in which the design matrix is defined by a Monte Carlo simulation. More efficient designs can be 
obtained with fewer runs when the objective is input-output effect analysis. Reference [12] describes, among others, the design of Taguchi orthogonal arrays and reference [18] addresses the design of experiments in conjunction with surrogate modeling. After the physical experiments or numerical simulations have been performed at the specified design

points, the $\mathrm{R}^{2}$ statistics can be calculated from equation (4) for various linear or higherorder effects. Effects associated with large $\mathrm{R}^{2}$ statistics explain the output variability more than the others and they must be accounted for to best-fit surrogate models such as equation (2). In the following, an example of effect analysis is presented that illustrates the characterization of a transient structural response using a Taguchi array design.

\subsection{Example: Large Scale Modeling of a Shock Response}

Traditionally, quantifying shock transmission through complex, jointed structures has been possible only with experimental methods. These experiments are expensive and time-consuming and thus only a few cases can be studied. With the advent of large scale computing capabilities, estimation of the shock transmission with numerical models is becoming a tractable problem.

The test article consists of several components fabricated from a variety of materials (titanium, 6061-T6 aluminum, 7075-T4 aluminum, SS-304 stainless steel and carbon steel). Figure 6 illustrates the individual components of the test article and a detail of the finite element mesh developed for simulating the response of the system to an explosive load. The components are connected to the center titanium mount with threaded, bolted and tapered tape joints. The purpose of the numerical simulation is to predict the acceleration levels witnessed by the upper and lower mass simulators. A series of physical experiments are also performed to measure the actual response of the system and assess the predictive accuracy of the numerical model. Making this prediction assumes that the transmission of the shock wave through the threaded assembly shown in Figure 6 is captured with accuracy. What makes it difficult, however, is that the energy dissipation characteristics of material interfaces are not known precisely.

In the numerical simulation, this uncertainty is represented by twelve random preload and friction coefficients. It is further assumed that four of the random input parameters take two values while the other eight take three values. Therefore, the total number of computer runs required to characterize fully the input space is $2^{4} \times 3^{8}=104,976$. Performing a full factorial analysis is currently impossible because a single evaluation of the model requires four to six hours of CPU time using 504 processors of the Los Alamos 3 Tera-Ops supercomputer. To limit the required simulation time, a subset of 48 runs is completed from parameter samples selected using the Taguchi orthogonal array technique. This particular design is adopted to eliminate aliasing, which refer to the confounding of linear effects (such as $\mathbf{a}_{\mathbf{i}}$ ) with second order or higher effects (such as $\mathbf{B}_{\mathbf{i j}}$ ). Because the transmission of shock across the mount to the payload components is the primary event of interest, statistical moments of the time history, shock response spectrum and power 
spectral density at six locations are used as features. Hence, a total of 36 features (2 moments x 3 outputs x 6 locations) are extracted for each one of the 48 runs. For each feature, a linear statistical model is constructed by ignoring higher-order effects $\boldsymbol{B}_{\mathrm{ij}}$ in equation (2). A summary of the $\mathrm{R}^{2}$ analysis is presented in Figure 7. 


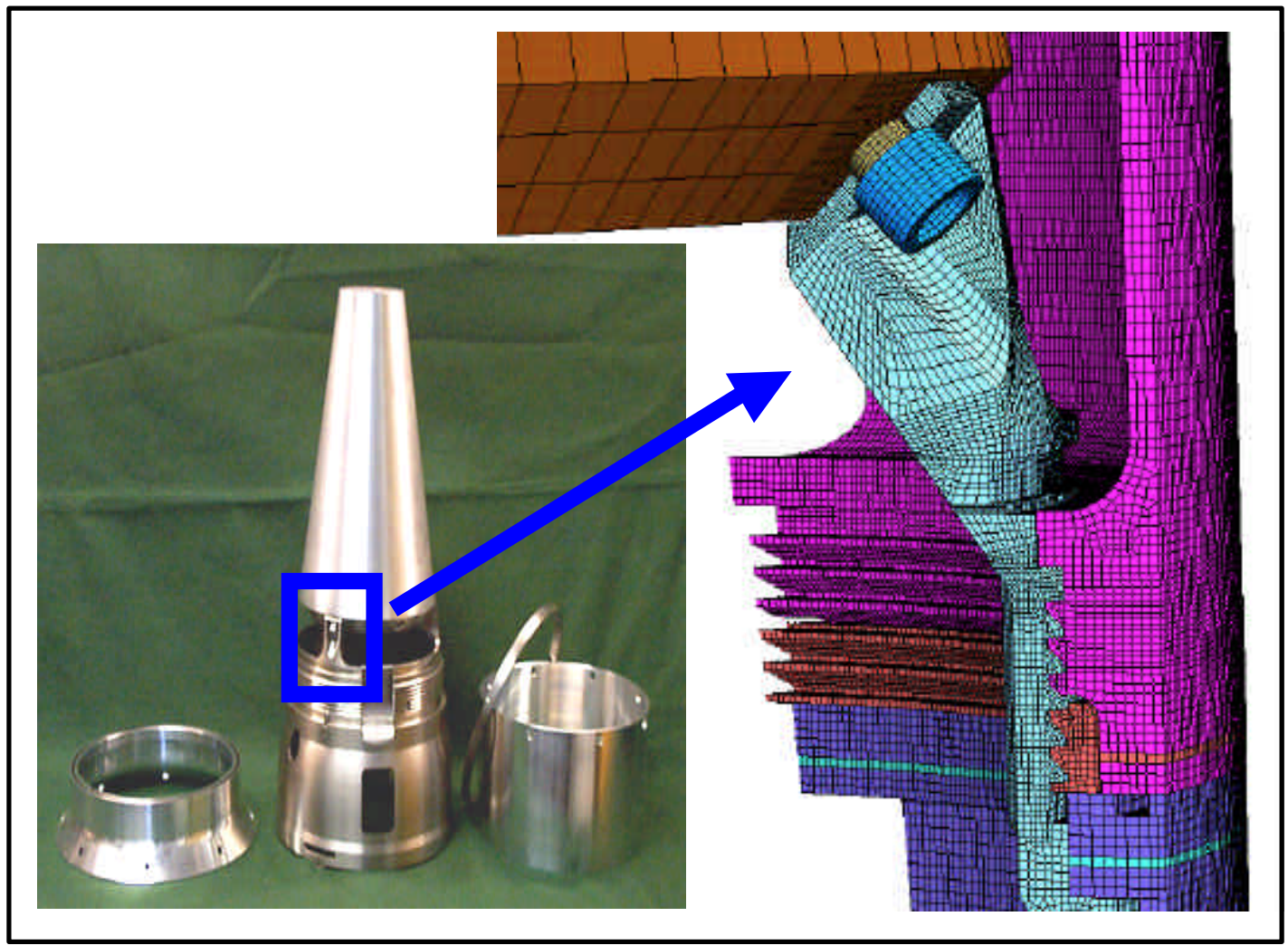

FIGURE 6. Components of the System and Detail of the Computational Mesh. (Left: Lower shell, titanium mount, upper mass simulator, retaining nut and lower shell. Right: Detail of the computational mesh showing the threaded interface between the upper and lower shells, retaining nut and titanium mount.)

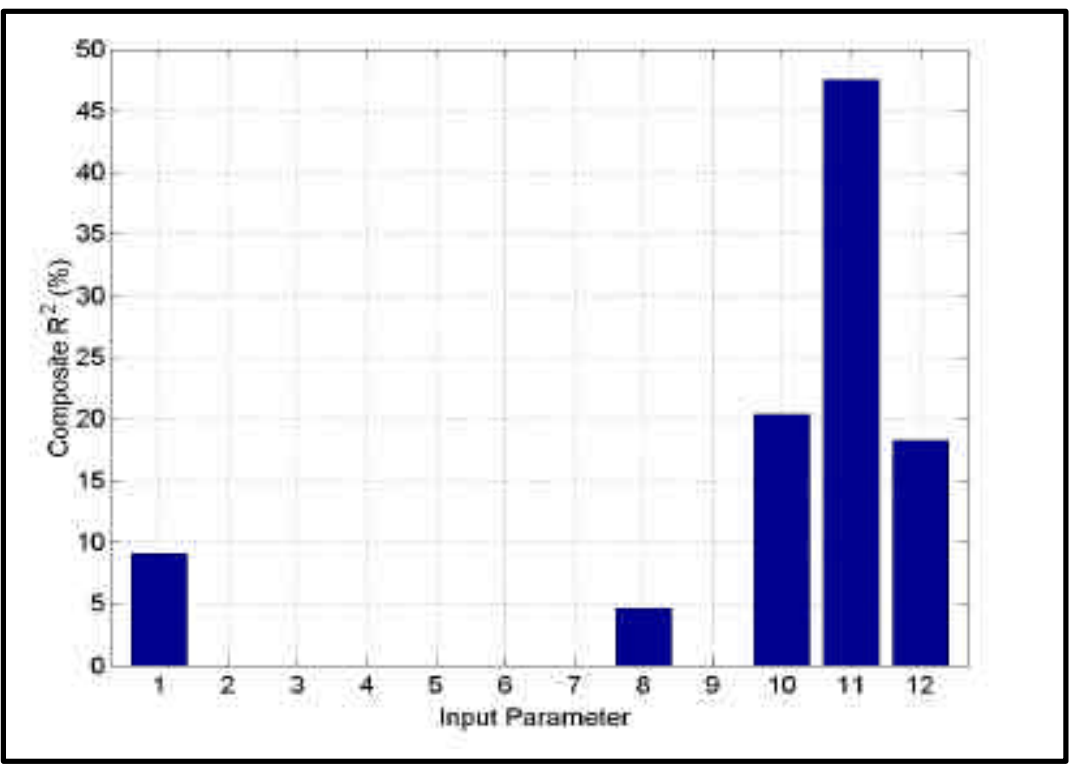

FIGURE 7. Input Parameters Most Responsible For the Output Variability. (Composite $R^{2}$ statistics for 36 features. The computer experiment is designed for the screening of linear effects using an orthogonal Taguchi array with 48 runs.) 
Figure 7 illustrates that the 36 features investigated exhibit global sensitivity to the five parameters $\mathbf{p}_{\mathbf{1}}, \mathbf{p}_{\mathbf{8}}, \mathbf{p}_{\mathbf{1 0}}, \mathbf{p}_{\mathbf{1 1}}$ and $\mathbf{p}_{\mathbf{1 2}}$. It demonstrates the significant reduction in dimensionality that can be achieved through parameter effect analysis. This model validation experiment is further discussed in references [6] and [19].

\section{TOWARDS THE ASSESSMENT OF TOTAL UNCERTAINTY}

This publication overviews the issues of uncertainty assessment for structural dynamics simulations and applications such as structural health monitoring. The propagation of parametric variability through numerical simulations is discussed. Parameter effect analysis is introduced as a practical method for identifying surrogate models and providing to the analysts valuable insight regarding the input-output relationship in the case of complex numerical models.

The inverse propagation of uncertainty is not illustrated here but numerous examples of parameter calibration, finite element model updating and other inference problems can be found in the literature. Inverse propagation of uncertainty refers to the inference of probability information for the control parameters of a physical experiment or input parameters of a numerical simulation, given observed uncertainty in the output. Among the possible frameworks, Bayesian inference is a choice commonly encountered in many disciplines of physics and engineering sciences. An example of Bayesian inference in the presence of uncertainty is provided in reference [20] that reports on the inference of a model of criticality in a nuclear physics experiment.

Uncertainty assessment is also a critical component of model validation. Model validation is formally defined as the substantiation that a model within its domain of applicability possesses a satisfactory range of accuracy consistent with the intended applications of the model. A pre-requisite to model validation is therefore that the total error between physical observation and model prediction be characterized. The approach generally agreed upon consists of breaking down the total error into individual components and estimating the probability information of each one. Equation (5) illustrates a total uncertainty model commonly adopted:

$$
\begin{aligned}
& \mathbf{y}_{\text {Measured }}=y_{\text {Predicted }}+\mathbf{e} \\
& \mathbf{y}_{\text {Predicted }}=\mathbf{M}\left(\mathbf{p}_{1} ; \mathbf{p}_{2} ; \cdots ; \mathbf{p}_{\mathrm{N}}\right) \\
& \mathbf{e} \sim \mathrm{N}\left(\mathbf{0} ; \mathbf{s}_{\text {Total }}\right)
\end{aligned}
$$

The total error is defined as the difference between measured and predicted responses. The numerical model $\mathbf{M}$ is a black box that provides an output $\mathbf{y}$ given $\mathbf{N}$ input parameters labeled $\mathbf{p}_{\mathbf{1}}$ through $\mathbf{p}_{\mathbf{N}}$. In addition, a probabilistic error model must be chosen. 
It consists in equation (5) of an unbiased Gaussian probability density function with unknown standard deviation $\mathbf{s}$ Total.

Characterizing the predictability of the numerical model can be achieved by decomposing the total error into independent contributions. For example, if the error is thought to be a Gaussian process that includes measurement error, parametric variability and discretization error, then the total variance can be decomposed into:

$$
S_{\text {Total }}^{2}=S_{M}^{2}+\sum_{j=1 \cdots N} S_{j}^{2}+S_{D}^{2}+S_{R}^{2}
$$

where $\mathbf{s}_{\mathbf{M}}$ denotes the standard deviation of measurement error, $\mathbf{s}_{\mathbf{j}}$ denotes the standard deviation of parametric variability for the $\mathrm{j}^{\text {th }}$ parameter $\mathbf{p}_{\mathbf{j}}$ and $\mathbf{s}_{\mathbf{D}}$ represents the discretization error. It may happen that the sources of uncertainty are not independent from each other, in which case the right-hand side in equation (6) becomes an upper bound. Variance decomposition is further complicated in the case of multivariate statistics because each variance term $\mathbf{s}^{2}$ in equation (6) then becomes a covariance matrix [S]. This happens when several, possibly correlated, output features $\left\{\mathbf{y}_{1} ; \mathbf{y}_{2} ; \ldots ; \mathbf{y}_{\mathrm{N}}\right\}$ are considered simultaneously.

In a typical model validation experiment, the total variance $\mathbf{s}_{\text {Total }}$ is obtained from a comparison of measured and predicted responses for a design of experiments that attempts to explore the input space as much as possible. Components such as $\mathbf{s}_{\mathbf{M}}$ and $\mathbf{s}_{\mathbf{D}}$ are estimated by investigating the measurement system and mesh convergence properties, respectively. The variability $\mathbf{s}_{\mathbf{j}}$ of the output due to input uncertainty of the $\mathrm{j}^{\text {th }}$ parameter is typically identified through an input-output effect analysis such as previously illustrated in section 3. In equation (6), the only term that remains unknown, $\mathbf{s}_{\mathbf{R}}$, represents the residual sources of uncertainty. They include model form error. Obtaining an estimation of model form error is critical to assess the validity of the numerical model over its domain of applicability. Once available, the probability information $\mathbf{N}\left(\mathbf{0} ; \mathbf{s}_{\mathbf{R}}\right)$ can be propagated to future predictions to assess confidence bounds associated with a prediction of the model. This may be the ultimate goal of total uncertainty assessment and model validation. Reference [21] develops a Bayesian framework for a similar propagation of uncertainty and reference [22] discusses an application of this methodology to the problem of radar range tracking in multiple and diffuse target situations.

\section{REFERENCES}

1. Garcia, J., 2001. "The Need for Computational Model Validation," Experimental Techniques, Vol. 25, No. 2, pp. 31-33.

2. Ren, W.-X., Harik, I.E., Lenett, M., Baseheart, T., 2001. "Modal Properties of the Roebling Suspension Bridge, FEM Modeling and Ambient Testing," $19^{\text {th }}$ SEM International Modal Analysis Conference (IMAC-XIX), Kissimmee, FL, February 5-8, 2001, pp.1139-1145. 
3. McCallen, B.D., Astaneh-Asl, A., 1998. "Computational Simulation of the Nonlinear Response of Suspension Bridges," Structural Engineers World Congress, San Francisco, CA, July 18-23, 1998. Report UCRL-JC-128901, Lawrence Livermore National Laboratory, Livermore, CA, October 1997, http://www.llnl.gov/tid/lof/documents/pdf/232213.pdf.

4. Doebling, S.W., Farrar, C.R., Prime, M.B., Shevitz, D.W., 1996. "Damage Identification and Health Monitoring of Structural and Mechanical Systems From Changes in Their Vibration Characteristics: A Literature Review," Report LA-13070-MS, Los Alamos National Laboratory, Los Alamos, NM, May 1996.

5. Sohn, H., Farrar, C.R., 2000. "Time Series Analysis for Locating Damage Sources in Vibration Systems," $25^{\text {th }}$ International Conference on Noise and Vibration Engineering (ISMA-25), Leuven, Belgium, September 13-15, 2000, pp. 187-191.

6. Hemez, F.M., Doebling, S.W., 2001. "Uncertainty, Validation of Computer Models and the Myth of Numerical Predictability," $2^{\text {nd }}$ European COST-F3 Conference on Structural System Identification, Kassel, Germany, September 5-7, 2001, to appear.

7. Proceedings of the $3^{\text {rd }}$ International Symposium on Sensitivity Analysis of Model Output (SAMO), 2001. Edited by P. Prado, Madrid, Spain, June 18-20, 2001.

8. Yager, R.R., Kacprzyk, J., Fedrizzi, M., 1996. Advances in the Dempster-Shafer Theory of Evidence, John Wiley \& Sons, New York.

9. Dumitrescu, D., Jain, L.C., Lazzerini, B., 2000. Fuzzy Sets and Their Application to Clustering and Training, International Series on Computational Intelligence, CRC Press, New York.

10. Ben-Haim, Y., Cogan, S., Sanseigne, L., 1998. "Usability of Mathematical Models in Mechanical Decision Processes," Mechanical Systems and Signal Processing, Vol. 12, No. 1, pp. 121-134.

11. McKay, M.D., Beckman, R.J., Conover, W.J., 1979. "A Comparison of Three Methods for Selecting Values of Input Variables in the Analysis of Output From a Computer Code," Technometrics, Vol. 21, No. 2, pp. 239-245.

12. Hedayat, A.S., Sloane, N.J.A., Stufken, J., 1999. Orthogonal Arrays: Theory and Applications, Springer-Verlag, New York.

13. Cukier, R.I., Schaibly, J.H., Shuler, K.E., 1975. "Study of the Sensitivity of Coupled Reaction Systems to Uncertainties in Rate Coefficients," Journal of Chemical Physics, Vol. 63, pp. 1140-1149.

14. Abaqus ${ }^{\mathrm{TM}} /$ Explicit User's Manual, 1998. Hibbitt, Karlsson \& Sorensen, Pawtucket, RI.

15. Lutz, M., 1996. Programming Python, O’Reilly \& Associates, Sebastopol, CA.

16. NESSUS User's Manual, 1996. Southwest Research Institute, San Antonio, TX.

17. Wojtkiewicz, S.F., Eldred, M.S., Field, R.V., Jr., Urbina, A., Red-Horse, J.R., "A Toolkit for Uncertainty Quantification in Large Computational Engineering Models," 42 ${ }^{\text {nd }}$ AIAA/ ASME/ASCE/AHS/ASC Structures, Structural Dynamics and Materials Conference, Seattle, WA, April 16-19, 2001, pp. 1-11.

18. Myers, R.H., Montgomery, D.C., 1995. Response Surface Methodology: Process and Product Optimization Using Designed Experiments, Wiley Inter-science, New York, NY.

19. Schultze, J.F., Hemez, F.M., Doebling, S.W., Butler, T.A., 2001. "A Novel Statistical Based Approach to Nonlinear Model Updating Using Response Features," SEM Annual Conference \& Exposition on Experimental and Applied Mechanics, Portland, Oregon, June 46, 2001, pp. 309-313.

20. Hanson, K.M., Booker, J., 2000. "Inference From Rossi Traces," Los Alamos Uncertainty Quantification Working Group, Los Alamos National Laboratory, Los Alamos, NM, September 2000, http://www.lanl.gov/home/kmh/.

21. Kennedy, M.C., O'Hagan, A., 2001, "Bayesian Calibration of Computer Models," Journal of the Royal Statistical Society (Series B), to appear, http://www.shef.ac.uk/ st1ao/.

22. Fraedrich, D., Gover, R., 2001. "Statistical Uncertainty Analysis as a Tool for Model Validation," $3^{\text {rd }}$ International Symposium on Sensitivity Analysis of Model Output (SAMO), Madrid, Spain, June 18-20, 2001. 